\title{
Attitudes of medical workers in China toward artificial intelligence in ophthalmology: a comparative survey
}

Bo Zheng ${ }^{1,2}$, Mao-nian Wu ${ }^{1,3}$, Shao-jun Zhu ${ }^{1,2}$, Hong-xia Zhou ${ }^{1,2,3}$, Xiu-lan Hao ${ }^{1,2}$, Fang-qin Fei ${ }^{4}$, Yun Jia ${ }^{5}$, Jian Wu ${ }^{6}$, Wei-hua Yang ${ }^{7 *}$ and Xue-ping Pan $^{8}$

\begin{abstract}
Background: In the development of artificial intelligence in ophthalmology, the ophthalmic Al-related recognition issues are prominent, but there is a lack of research into people's familiarity with and their attitudes toward ophthalmic Al. This survey aims to assess medical workers' and other professional technicians' familiarity with, attitudes toward, and concerns about Al in ophthalmology.

Methods: This is a cross-sectional study design study. An electronic questionnaire was designed through the app Questionnaire Star, and was sent to respondents through WeChat, China's version of Facebook or WhatsApp. The participation was voluntary and anonymous. The questionnaire consisted of four parts, namely the respondents' background, their basic understanding of Al, their attitudes toward Al, and their concerns about Al. A total of 562 respondents were counted, with 562 valid questionnaires returned. The results of the questionnaires are displayed in an Excel 2003 form.

Results: There were 291 medical workers and 271 other professional technicians completed the questionnaire. About 1/3 of the respondents understood Al and ophthalmic Al. The percentages of people who understood ophthalmic Al among medical workers and other professional technicians were about $42.6 \%$ and $15.6 \%$, respectively. About $66.0 \%$ of the respondents thought that Al in ophthalmology would partly replace doctors, about $59.07 \%$ having a relatively high acceptance level of ophthalmic Al. Meanwhile, among those with Al in ophthalmology application experiences (30.6\%), above $70 \%$ of respondents held a full acceptance attitude toward $\mathrm{Al}$ in ophthalmology. The respondents expressed medical ethics concerns about Al in ophthalmology. And among the respondents who understood Al in ophthalmology, almost all the people said that there was a need to increase the study of medical ethics issues in the ophthalmic Al field.

Conclusions: The survey results revealed that the medical workers had a higher understanding level of Al in ophthalmology than other professional technicians, making it necessary to popularize ophthalmic Al education among other professional technicians. Most of the respondents did not have any experience in ophthalmic Al but generally had a relatively high acceptance level of Al in ophthalmology, and there was a need to strengthen research into medical ethics issues.
\end{abstract}

\footnotetext{
* Correspondence: benben0606@139.com

${ }^{7}$ Affiliated Eye Hospital of Nanjing Medical University, No.138 Hanzhong

Road, Gulou District, 210029 Nanjing, Jiangsu, China

Full list of author information is available at the end of the article
}

C C The Author(s). 2021 Open Access This article is licensed under a Creative Commons Attribution 4.0 International License, which permits use, sharing, adaptation, distribution and reproduction in any medium or format, as long as you give appropriate credit to the original author(s) and the source, provide a link to the Creative Commons licence, and indicate if changes were made. The images or other third party material in this article are included in the article's Creative Commons licence, unless indicated otherwise in a credit line to the material. If material is not included in the article's Creative Commons licence and your intended use is not permitted by statutory regulation or exceeds the permitted use, you will need to obtain permission directly from the copyright holder. To view a copy of this licence, visit http://creativecommons.org/licenses/by/4.0/ The Creative Commons Public Domain Dedication waiver (http://creativecommons.org/publicdomain/zero/1.0/) applies to the data made available in this article, unless otherwise stated in a credit line to the data. 
Keywords: Artificial intelligence, Ophthalmic artificial intelligence, Artificial intelligence in ophthalmology, Artificial intelligence in medicine, Replacing doctors, Doctor replacement, Medical ethics

\section{Background}

In recent years, with the increase of computational speed, neural networks have regained prosperity after hitting its rock bottom. The deep convolutional neural network AlexNet [1] winning the first place in the ImageNet competition has led to the rapid development of deep learning technology. After that, deep learning network models [210] have emerged generation after generation, which has accelerated the development of artificial intelligence (AI) technology. Through AI, researchers can make the preliminary diagnosis of skin cancers, achieve rapid intraoperative diagnosis of brain tumors, diagnose 55 common diseases in pediatrics based on electronic medical records in Chinese, identify rare genetic diseases through facial photographs, and generate the findings that early and frequent patient movements can reduce the risk of postintensive care syndrome and long-term dysfunction after analyzing patients' movement activities in the intensive care units. [11-15]

Medical instruments are frequently used in clinical examinations in modern medicine, and imaging equipment is an important part. Imaging equipment is used for X-ray imaging, magnetic resonance imaging, ultrasound imaging, tomography imaging, and fundus photography, etc. The data in the ophthalmology field is diverse and huge, among which the most common types are fundus photographs and optical coherence tomography (OCT) images, making it the most extensively researched field with AI. Fundus photography and OCT are regular examinations used in ophthalmology, through which a vast amount of high-quality standard images can be obtained. These images are suitable for analysis and process by AI deep learning technology to further assist doctors in diagnosing ophthalmopathies. Using deep learning technology in AI, Google researchers have created an algorithm that can detect diabetic retinopathy and macular edema. By analyzing human retinal images, this algorithm can very accurately predict risk factors affecting cardiovascular health. $[16,17]$ The researchers from Sun Yat-sen University in China have developed a deep learning model called CC-Cruiser for recognizing congenital cataract, which is also able to diagnose blinding diseases such as age-related macular degeneration and diabetic macular edema after trained with deep learning algorithms based on OCT images [18, 19]. Besides, there are related studies that use AI technology for the segmentation of ophthalmic images [20, 21], and the classification of ophthalmopathies [22-24], etc.

Artificial intelligence technology has been studied so extensively in ophthalmology that some even have been on the commercializing stage [25], thus leading to some people believing that AI may be able to replace doctors. Some researchers investigated the attitudes of medical undergraduates to the application of AI in radiology and medicine [26], the attitudes of medical students in other regions to AI [27], the influence of artificial intelligence on radiology [28], as well as how to cope with the ethical challenges in medical AI $[29,30]$, etc. However, few people know about medical workers' familiarity with and their attitudes toward AI in ophthalmology. For this purpose, a questionnaire was designed to assess medical workers' (health care workers or medical students) understanding level of and their attitudes toward AI. Meanwhile, the questionnaire also surveyed other professional technicians (engineers, teachers, technicians, experimenters, etc. in non-medical fields) using the same questions as a comparison.

The survey of AI in ophthalmology-related questions cannot only evaluate the attitudes of medical workers (health care workers or medical students) and other professional technicians toward AI and clarify the dilemma facing the current technological development; it can also provide theoretical guidance for its future practice and application. At present, in the development of AI in ophthalmology, there are a lot of existing and foreseeable medical ethical problems. For example, the medical responsibilities are unclear, the problem of the security of patient privacy data and the risk of the weakening of the doctor's professional status, etc. Analysis of the causes of these problems through this survey may help to put forward corresponding countermeasures so that we can draw on advantages and avoid disadvantages in the future development of $\mathrm{AI}$ in ophthalmology.

\section{Methods}

Using the Questionnaire Star APP (a professional questionnaire survey app in China, easy to edit and distribute survey questionnaires), we designed an electronic questionnaire that consisted of four parts. The first part was the respondent's basic information, including the respondent's sex, age, educational level, place of residence, work area and professional title; the second part was about the respondent's basic understanding of $\mathrm{AI}$, including whether the respondent understood $\mathrm{AI}$, medical $\mathrm{AI}$, and $\mathrm{AI}$ in ophthalmology, as well as the respondent's evaluation of the current development of $\mathrm{AI}$ in ophthalmology; the third part was about the respondent's attitude to AI, including whether he/she thought AI in ophthalmology would replace doctors, whether he/she 
had experience in AI in ophthalmology application, and his/her acceptance level of AI in ophthalmology; and the fourth part was about the respondent's concerns about AI, which included the respondent' specific concerns about AI in ophthalmology and whether he/she thought it was necessary to strengthen medical ethics research in the field.

According to the pre-investigation experience and the principle of the rank order scale, the basis for the classification of the four ranks is determined. There were 4 grading used to reflect the basic understanding of AI in the second part of the electronic questionnaire. The grading "Completely" means the understanding level of $\mathrm{AI} \geq 90 \%$; The grading "Almost" means the understanding level of AI between $50 \%$ and $89 \%$ (include $50 \%$ and $89 \%)$; The grading "A little" means the understanding level of AI between $10 \%$ and $49 \%$ (include $10 \%$ and $49 \%)$; The grading "Not understand" means the understanding level of $\mathrm{AI} \leq 9 \%$.

There were 4 grading used to reflect the respondent's attitude to AI in the third part of the electronic questionnaire. The grading "Completely" means the acceptance level of ophthalmic AI $\geq 80 \%$; The grading "Partly" means the acceptance level of ophthalmic AI between $20 \%$ and $79 \%$ (include $20 \%$ and $79 \%$ ); The grading "No" means the acceptance level of ophthalmic AI $\leq$ $19 \%$; The grading "Don't understand" means the respondents didn't understand the question.

Respondents of the questionnaire were mainly members of the Zhejiang Society of Mathematical Medicine, with their locations covering various cities and counties mainly in Zhejiang Province. They worked as ophthalmologists, medical students, AI technicians, and professional technicians in other fields. Their educational levels were above junior high school and could understand the questionnaire well. The questions in the questionnaire have been investigated, sorted and summarized repeatedly in a broad and deep way. The survey is a targeted group survey. Questionnaires were sent to medical workers through professional ophthalmological or medical intelligence groups and other professionals through related professional groups. Before we collect the questionnaires for statistical analysis, each respondent was invited to fill out the questionnaire once based on a voluntary and anonymous principle and was informed that the results of the survey would be further used for statistical assessment and publication.

This study is an epidemiological survey research design, containing 15 questions, and usually requires a sample size of at least 5-10 times the number of questions, usually about 15 times. Therefore, the sample we selected is between 15 and 20 times the number of questionnaires, with 291 medical workers and 271 other professional technicians. This study mainly uses statistical analysis, chi-square analysis and odds ratio analysis. The relevant indicators include statistical analysis results, chi-square value, odds ratio (OR) value, Probability $(\mathrm{P})$ value and $95 \% \mathrm{CI}$. A total of 562 respondents were counted, with 562 valid questionnaires returned. The results of the questionnaires are displayed in an Excel 2003 form.

According to the Article 3 of the Measures for the Ethical Review of Biomedical Research Involving Humans issued by the National Health and Family Planning Commission in 2016, ethical review is unnecessary for the study.

\section{Results}

\section{Basic information of respondents}

Of the 562 respondents, 291 were medical workers $(51.8 \%)$ with the rest being other professional technicians $(48.2 \%)$. As shown in Table 1, nearly half of them were from prefecture-level cities (47.7\%), about $1 / 4$ (24.9\%) were from provincial capital cities, and the remaining were from other regions $(27.4 \%) .10 .0 \%$ had

Table 1 Respondents' basic information $(N=562)$

\begin{tabular}{ll}
\hline Characteristic & $\begin{array}{l}\text { Respondents } \\
\mathbf{n}(\%)\end{array}$ \\
\hline Sex & \\
Male & $216(38.4)$ \\
Female & $346(61.6)$ \\
Age (years) & \\
25 or less & $70(12.5)$ \\
$25-45$ & $340(60.5)$ \\
45 or more & $152(27.1)$ \\
Work area & \\
Medical worker (health care worker or medical student) & $291(51.8)$ \\
Other professional technicians & $271(48.2)$ \\
Place of residence & \\
Provincial capital & $140(24.9)$ \\
Prefecture-level city & $268(47.7)$ \\
County and below & $152(27.1)$ \\
Abroad & $2(0.4)$ \\
Education & \\
Doctor and above & \\
Master & $56(10.0)$ \\
Bachelor & $73(13.0)$ \\
Other lower education & $244(43.4)$ \\
Primgraded & $189(33.6)$ \\
Intermediate & \\
\hline & $180(32.0)$ \\
\hline & $143(25.4)$ \\
\hline & $177(31.5)$ \\
\hline
\end{tabular}


a doctor's degree or higher education, $13.0 \%$ had a master's degree, and $77.1 \%$ had a bachelor's degree or lower education; $31.5 \%$ had senior titles, $25.4 \%$ were with intermediate titles, and $32.0 \%$ were primary and ungraded professionals. The complex structure of these respondents had relatively good social representative value.

\section{Respondents' basic understanding and attitudes towards}

\section{Al}

In Table 2, the percentage of respondents who completely understood and almost understood AI was $37.9 \%$, who understood a little was $52.1 \%$, and who didn't understand AI was $10.0 \%$; the percentage of respondents who completely understood and almost understood medical AI was $31.7 \%$, who understood a little was $44.3 \%$ and who didn't understand medical AI was $24.0 \%$; the percentage of respondents who completely understood and almost understood AI in ophthalmology was $29.6 \%$, who understood a little was $34.5 \%$, and who didn't understand AI in ophthalmology was $35.9 \%$. That is to say, the proportion of respondents whose understanding level of AI, medical AI, and AI in ophthalmology was "completely", "almost" or "a little" was gradually decreasing in the mentioned order (AI,

Table 2 Respondents' basic understanding of artificial intelligence $(N=562)$

\begin{tabular}{ll}
\hline Understanding level & $\begin{array}{l}\text { Respondents } \\
\mathbf{n}(\%)\end{array}$ \\
\hline Artificial intelligence & \\
Completely & $39(6.9)$ \\
Almost & $174(31.0)$ \\
A little & $293(52.1)$ \\
Not understand & $56(10.0)$ \\
Medical artificial intelligence & \\
Completely & $33(5.9)$ \\
Almost & $145(25.8)$ \\
A little & $249(44.3)$ \\
Not understand & $135(24.0)$ \\
Ophthalmic artificial intelligence & \\
Completely & $34(6.1)$ \\
Almost & $132(23.5)$ \\
A little & $194(34.5)$ \\
Not understand & $202(35.9)$ \\
Current development of ophthalmic artificial intelligence \\
Very good \\
Good & $109(19.4)$ \\
Average & $240(42.7)$ \\
Poor & $198(35.2)$ \\
\hline & $15(2.7)$ \\
\hline
\end{tabular}

medical AI, and ophthalmological AI), while the proportion of people who did not know about AI, medical AI, and $\mathrm{AI}$ in ophthalmology was gradually increasing in the same order.

Among the respondents in Table 2, there were 19.4\% of them thought that the current development of AI in ophthalmology was very good. About $42.7 \%$ of respondents thought that its current development was good. There were $35.2 \%$ of respondents thought that its current development was average. As shown in Table 3, on the question of whether AI in ophthalmology would replace doctors, there were $24.0 \%$ of respondents said no. But $66.0 \%$ of respondents thought it would partly replace doctors. There were $69.4 \%$ of the respondents had no ophthalmic AI-related experience while the rest had applied or experienced AI in ophthalmology. There were $59.1 \%$ of the respondents had a relatively high acceptance level of AI in ophthalmology, with only $2.1 \%$ against it.

Therefore, the popularity of AI in China is relatively high, and the respondents had a certain understanding of artificial intelligence (such as alpha go, etc.), but there were still deficiencies in professional fields (such as ophthalmology). The most of respondents had a high degree of acceptance of AI in ophthalmology, and held a positive attitude towards its current development, believing that AI can improve the quality of people's lives. Although most respondents had no experience in ophthalmic AI, they still believed that ophthalmic AI would partially replace doctors. But doctors played a vital role

Table 3 Respondents' attitudes toward artificial intelligence $(N=562)$

\begin{tabular}{ll}
\hline Al in ophthalmology will replace ophthalmologists & $\begin{array}{l}\text { Respondents } \\
\mathbf{n}(\%)\end{array}$ \\
\hline Completely & $10(1.8)$ \\
Partly & $371(66.0)$ \\
Not & $135(24.0)$ \\
Don't understand & $46(8.2)$ \\
Do you have any experience with Al in & Respondents \\
ophthalmology? & $n(\%)$ \\
Being using or about to use & $29(5.2)$ \\
Having applied & $49(8.7)$ \\
Having experienced & $94(16.7)$ \\
Having no related experience & $390(69.4)$ \\
Acceptance level of Al in ophthalmology & $\mathrm{Respondents}$ \\
& $\mathrm{n}(\%)$ \\
Completely & $332(59.1)$ \\
Partly & $218(38.8)$ \\
Not accept & $10(1.8)$ \\
Strongly resist & $2(0.4)$ \\
\hline
\end{tabular}


Table 4 Medical workers and other professional technicians ' basic understanding of artificial intelligence ( $\mathrm{N}$ of medical workers = 291, N of other professional technicians $=271$ )

\begin{tabular}{|c|c|c|c|c|}
\hline Understanding level & Medical workers n(\%) & Other professional technicians,n(\%) & Chi-Square, $X^{2}$ & Asymp. Sig., $\mathrm{P}$ \\
\hline \multicolumn{5}{|l|}{ Artificial intelligence } \\
\hline Completely & $17(5.8)$ & $22(8.1)$ & 43.207 & 0 \\
\hline Almost & $121(41.6)$ & $53(19.6)$ & & \\
\hline A little & $140(48.1)$ & $153(56.5)$ & & \\
\hline Not understand & $13(4.5)$ & $43(15.9)$ & & \\
\hline \multicolumn{5}{|c|}{ Medical artificial intelligence } \\
\hline Completely & $18(6.2)$ & $15(5.5)$ & 92.059 & 0 \\
\hline Almost & 113(38.8) & $32(11.8)$ & & \\
\hline A little & $132(45.4)$ & 117(43.2) & & \\
\hline Not understand & 28(9.642) & 107(39.5) & & \\
\hline \multicolumn{5}{|c|}{ Ophthalmic artificial intelligence } \\
\hline Completely & 19(6.543) & $15(5.5)$ & 117.776 & 0 \\
\hline Almost & 105(36.1) & $27(10.0)$ & & \\
\hline A little & $121(41.6)$ & $73(26.9)$ & & \\
\hline Not understand & $46(15.8)$ & 156(57.6) & & \\
\hline
\end{tabular}

in the diagnosis and treatment of diseases and would not be completely replaced.

\section{Comparison of perceptions and attitudes towards Al} between medical workers and other professional technicians Table 4; Figs. 1 and 2, and Fig. 3 show that the proportion of medical workers whose understanding level was "completely understand" or "almost understand" was $47.4 \%, 45.0 \%$, and $42.6 \%$, respectively, for AI, medical $\mathrm{AI}$, and $\mathrm{AI}$ in ophthalmology. For other professional technicians, the proportion was $27.7 \%, 17.4 \%$, and $15.5 \%$, respectively. The Pearson Chi-Square of work area and understanding level for AI, medical AI, and AI in ophthalmology was 43.207, 92.059, and 117.776 respectively. All the $\mathrm{P}$ was 0 , which means the work area of the respondents was related to their understanding of AI, medical AI, and AI in ophthalmology. Table 5 was a simple version of Table 4. In Table 5, option "completely" and "almost" were merged together, option "a little" and "not understand" were merged together. As shown in Table 4, all the $\mathrm{P}$ were equal 0 , all the odds ratio (OR) were bigger than 1 and all the lower bound of the $95 \% \mathrm{CI}$ were bigger than 1 .

As shown in Figs. 4 and 5, about $71 \%$ of medical workers and $53 \%$ of other professional technicians believed that the current development of AI in

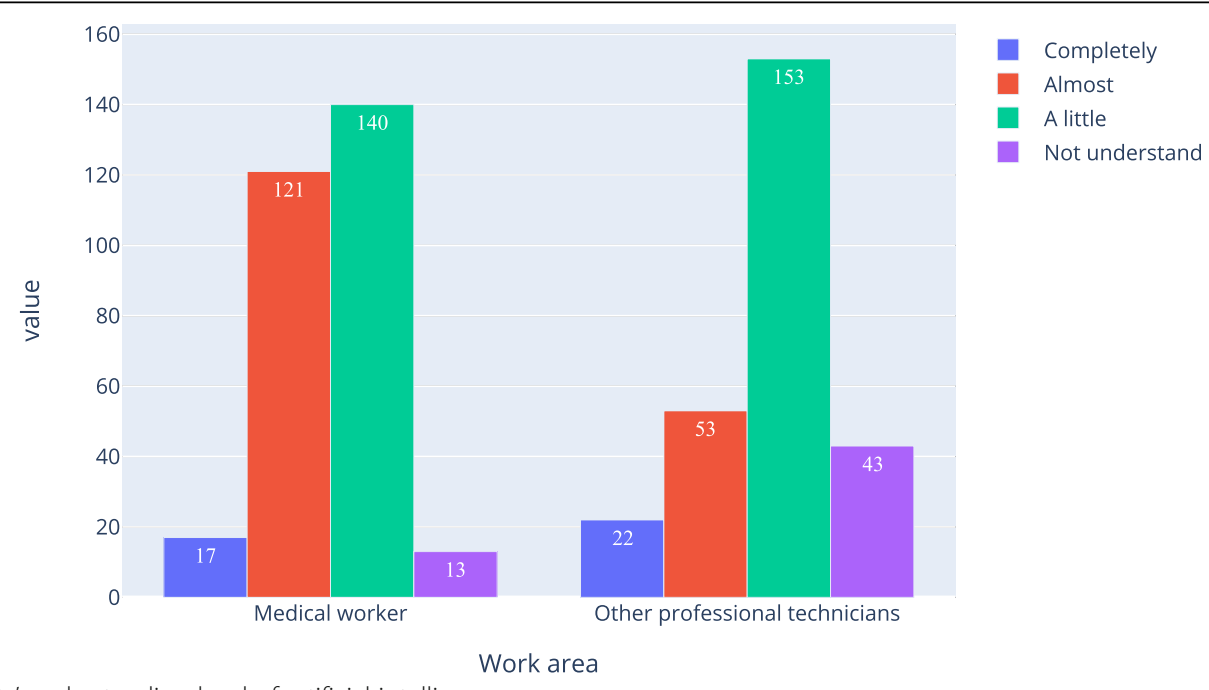

Fig. 1 Respondents' understanding level of artificial intelligence 


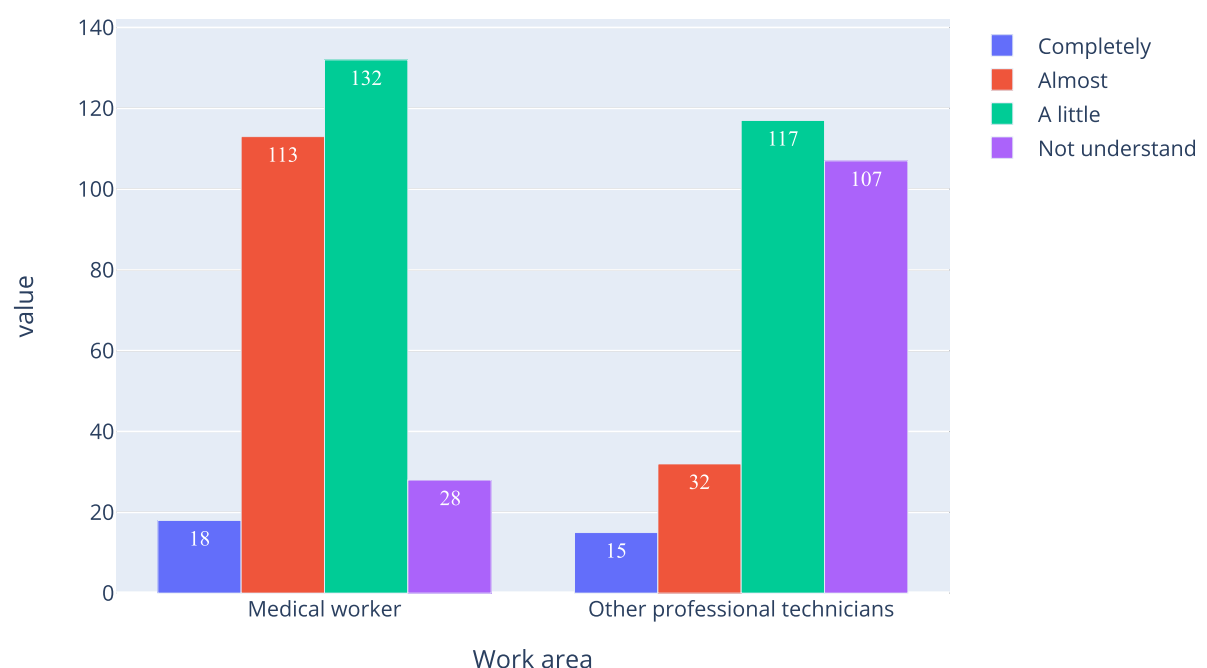

Fig. 2 Respondents' understanding level of medical artificial intelligence

ophthalmology was good; while among those who completely understood and almost understood AI in ophthalmology, about $81 \%$ of medical workers and $76 \%$ of other professional technicians thought that the current development of AI in ophthalmology was good.

For the two different groups by working fields, the respondents' understanding level of AI, medical AI, and AI in ophthalmology tended to drop in the mentioned order. The proportion of respondents whose understanding level of these three AIs was "completely understand" or "almost understand" was greater among medical workers than among other professional technicians. The close combination of medicine and AI had enabled medical workers to understand more about AI. Therefore, AI is relatively well popularized in the medical field; meanwhile, there is a need to enhance the popularization of artificial intelligence-related knowledge among people in other fields.

As shown in Figs. 6, 7 and 8, about $77.0 \%$ of medical workers and $57.9 \%$ of other professional technicians believed that AI in ophthalmology would completely or partly replace doctors. There were $56.4 \%$ of medical workers and $83.4 \%$ of other professional technicians had no experience in the application of AI in ophthalmology, while among those with experience in the application of AI in ophthalmology, there were $84.3 \%$ of medical workers and $73.3 \%$ of other professional technicians would fully accept it.x

In Table 6, option "completely" and "partly" were merged together, option "no" and "don't understand" were merged together, option "no" and "strongly resist" were merged together; for question "Do you have an

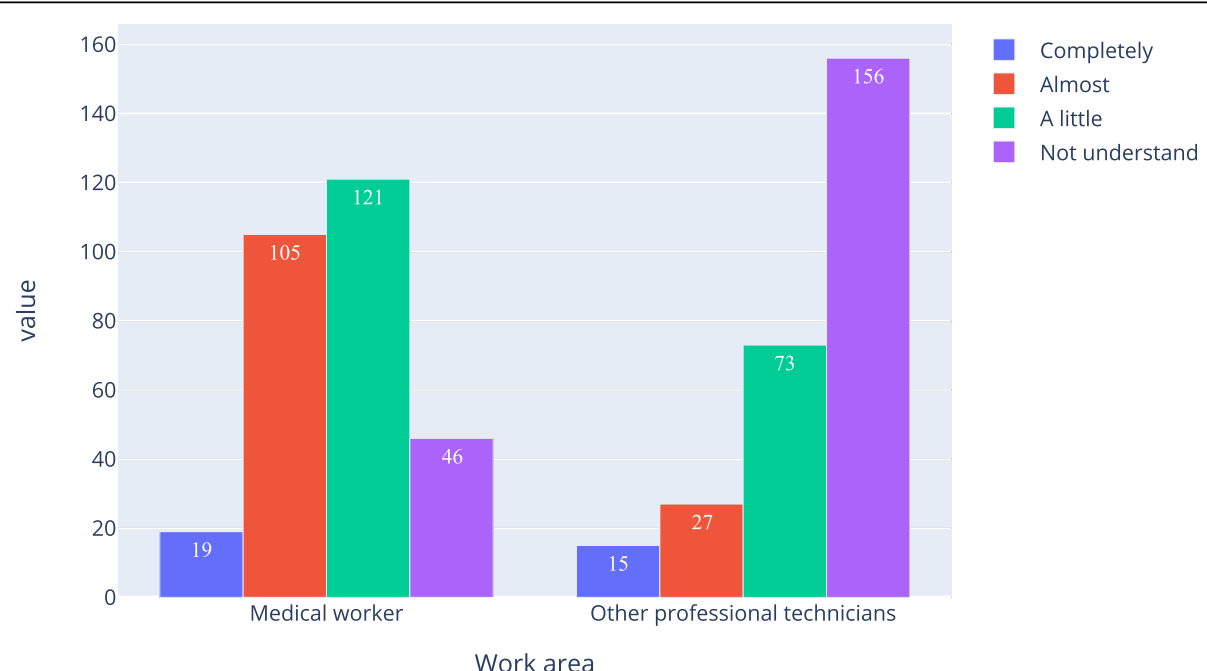

Fig. 3 Respondents' understanding level of ophthalmic artificial intelligence 
Table 5 Simple version of medical workers and other professional technicians ' basic understanding of artificial intelligence ( $\mathrm{N}$ of medical workers $=291, \mathrm{~N}$ of other professional technicians $=271$ )

\begin{tabular}{|c|c|c|c|c|c|c|}
\hline Understanding level & $\begin{array}{l}\text { Medical workers, } \\
\mathrm{n}(\%)\end{array}$ & $\begin{array}{l}\text { other professional } \\
\text { technicians, } \mathrm{n}(\%)\end{array}$ & Chi-Square, X2 & Asymp. Sig., P & OR & $95 \% \mathrm{Cl}$ \\
\hline \multicolumn{7}{|l|}{ Artificial intelligence } \\
\hline Completely and Almost & 138(47.4) & $75(27.7)$ & 23.249 & 0 & 2.357 & $1.658-3.351$ \\
\hline A little and Not understand & $153(52.6)$ & 196(72.3) & & & & \\
\hline \multicolumn{7}{|l|}{ Medical artificial intelligence } \\
\hline Completely and Almost & $131(45.0)$ & 47(17.4) & 49.658 & 0 & 3.902 & $2.642-5.764$ \\
\hline A little and Not understand & $160(55.0)$ & $224(82.7)$ & & & & \\
\hline \multicolumn{7}{|c|}{ Ophthalmic artificial intelligence } \\
\hline Completely and Almost & $124(42.6)$ & $42(15.5)$ & 49.564 & 0 & 4.048 & $2.706-6.056$ \\
\hline A little and Not understand & $167(57.4)$ & $229(84.5)$ & & & & \\
\hline
\end{tabular}

experience with an ophthalmic artificial intelligence application?", option "having no related experience" was divided into one group, other options were combined into another group. As shown in Table 6, the $\mathrm{P}$ of the first and second questions were equal 0 , the odds ratio (OR) of them were bigger than 1 and the lower bound of the $95 \% \mathrm{CI}$ of them were bigger than 1 . So the work area of the respondents was related to the two questions. The $\mathrm{P}$ of the third question was 0.061 , the OR was 2.357 but the $95 \% \mathrm{CI}$ was $0.883-12.312$. So it wasn't related to the work area of the respondents.

For the two groups by working fields, most of people did not have experience in applying artificial intelligence to ophthalmology, but still believe that AI will partially replace doctors, which shows that the respondents have a certain understanding of AI, but AI had not been widely used in ophthalmology. Compared with other professional technicians, medical workers had more experience in AI. AI was developing rapidly in ophthalmology and had better prospects. There was only $1.8 \%$ of the respondents believed that AI would completely replace ophthalmologists, indicating that people recognized the value of ophthalmologists and believed that even if AI developed better in the future, it is still only an aid to ophthalmologists rather than a replacement. At the same time, there was only $2.1 \%$ of the respondents had an unacceptable attitude towards AI in ophthalmology, indicating that people recognized the combination of $\mathrm{AI}$ and the ophthalmology. This recognition had nothing to do with the working fields, but people believed that AI could improve people's lives.

\section{Respondents' concerns about artificial intelligence}

In Table 7, among the respondents, $56.4 \%$ said that in the current ophthalmic AI practice, medical responsibilities are unclear; $49.3 \%$ said that the quality of AI in ophthalmology services was difficult to guarantee; while the percentage of those who thought there existed

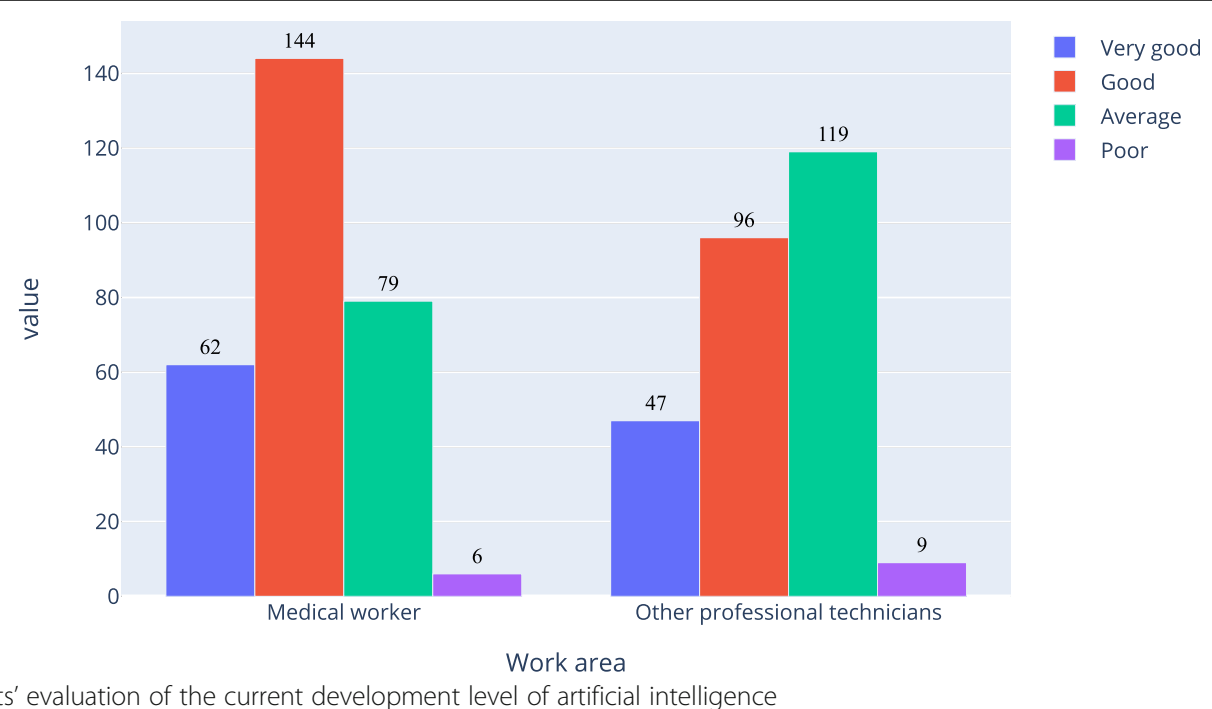

Fig. 4 Respondents' evaluation of the current development level of artificial intelligence 


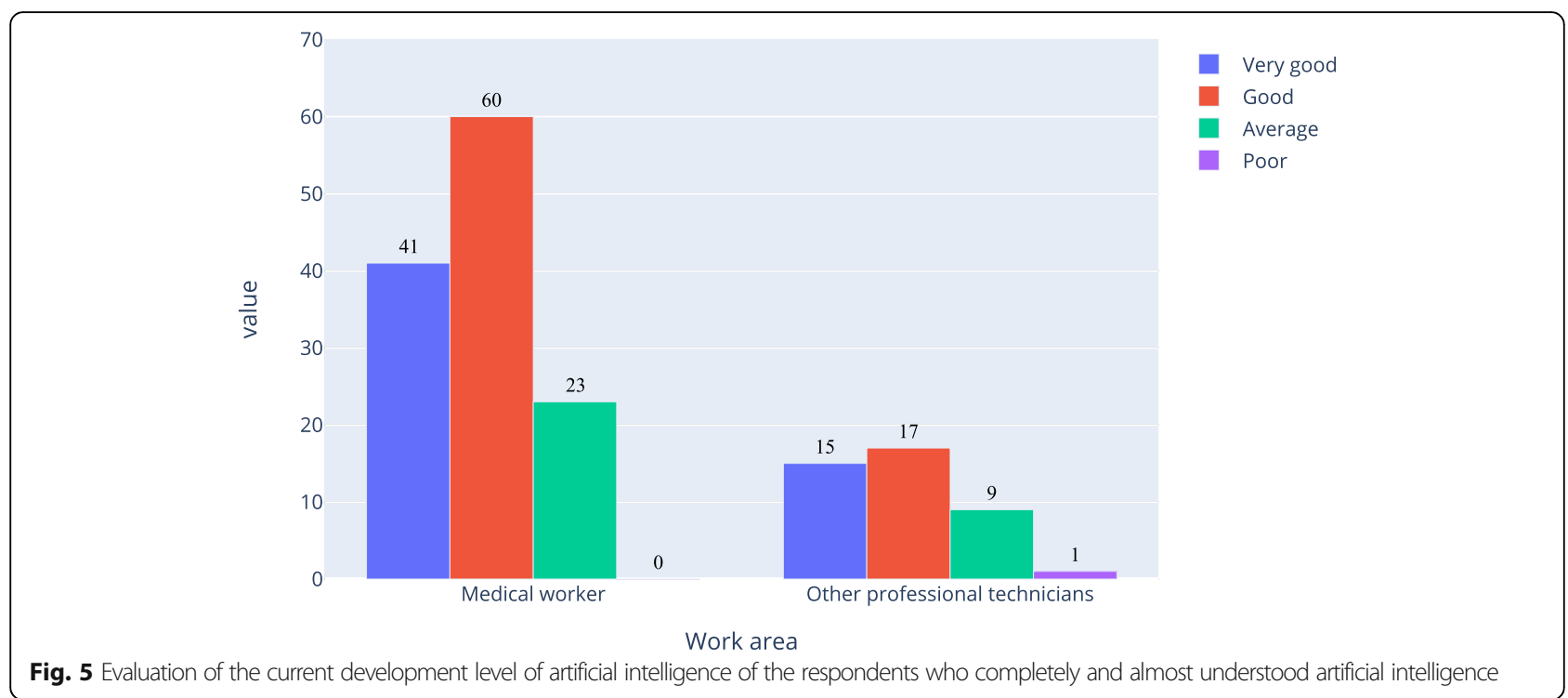

problems such as extreme high prices, medical ethical risks and lack of political support was about $40 \%$. More than $90 \%$ of the respondents thought there was a need to strengthen medical ethics research in the ophthalmic AI field. Among those who completely and almost understood AI in ophthalmology, $98.4 \%$ of medical workers and $95.2 \%$ of other professional technicians believed it was necessary to strengthen medical ethics research in the field, as shown in Fig. 9. This is enough to showcase the importance of addressing medical ethical issues in the ophthalmic AI field.

\section{Discussion}

Among the medical workers, there was $42.6 \%$ could understand ophthalmic AI, about $84.3 \%$ fully accepted ophthalmic AI, there was $43.6 \%$ had related applications experience in ophthalmic AI, there was $77 \%$ believed that ophthalmic AI would completely or partially replace doctors, and there was $98.4 \%$ believed that ophthalmic AI ethics research needs to be strengthened. These results help to make some targeted improvements in ophthalmic AI research, such as setting up more pilots so that people have more opportunities to experience ophthalmic AI and more accept ophthalmic AI, therefore, the application of ophthalmic AI can be promoted to the clinic. During diagnosis and treatment, the workload of medical workers is reduced.

In the survey, the proportion of medical workers was roughly equal to the proportion of other professional technicians. The proportion of respondents who did not understand $\mathrm{AI}$ among other professional technicians was about three times that of medical workers. And the

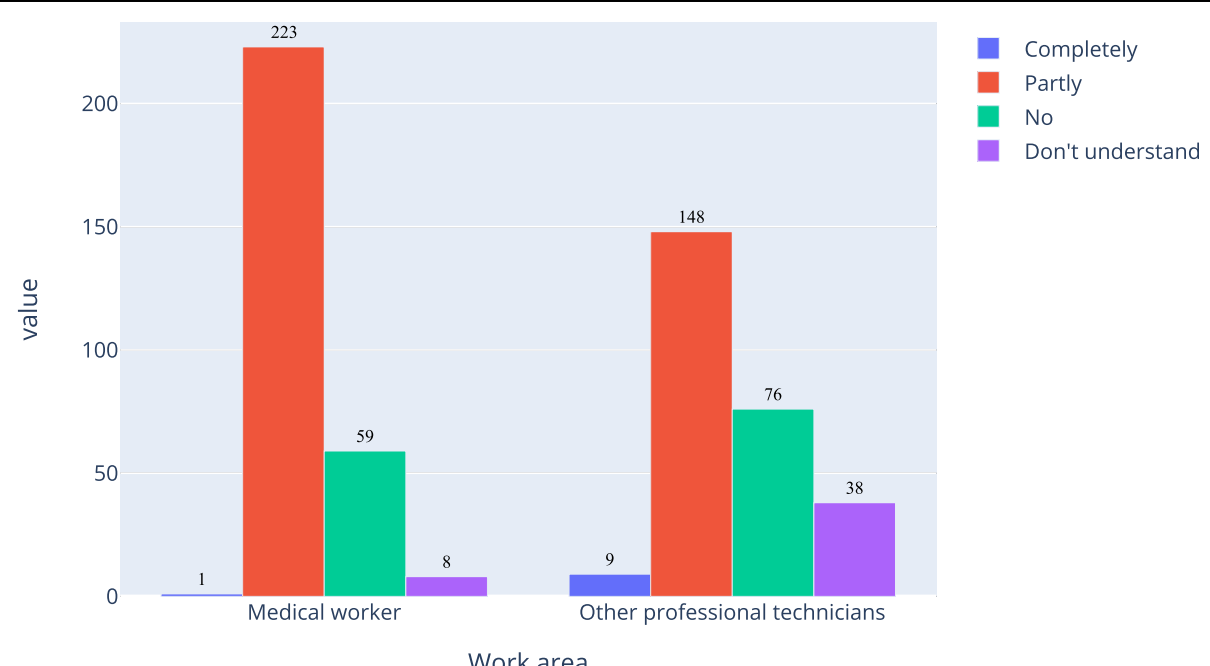

Work area

Fig. 6 Respondents' prediction on the extent to which ophthalmic artificial intelligence would replace doctors 


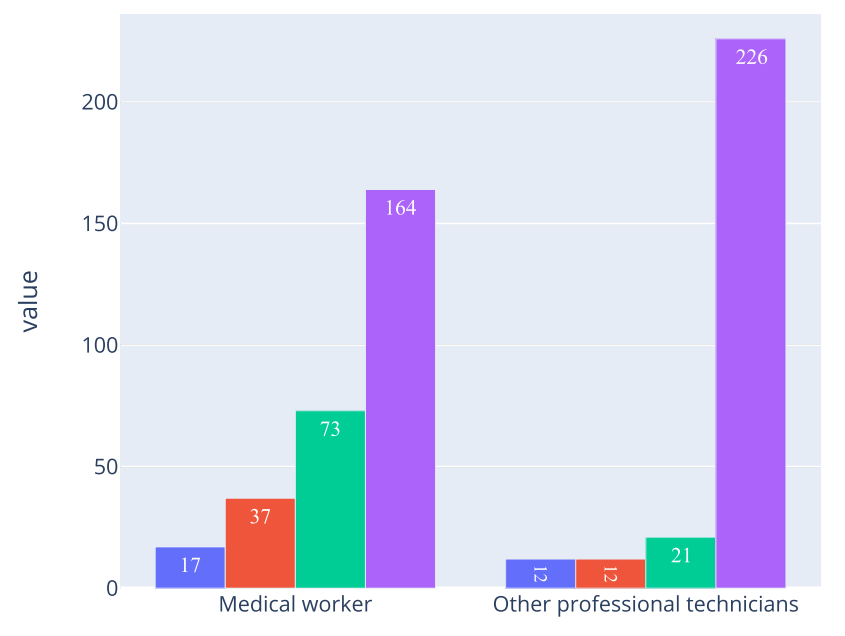

Being using or about to use

Having applied

Having experienced

Having no related experience

Work area

Fig. 7 Respondents' experience in the application of ophthalmic artificial intelligence

proportion of those who didn't understand medical AI among other professional technicians was about 4 times that of medical workers. The proportion of people who did not understand AI in ophthalmology among other professional technicians was about four times that of medical workers. Hence one can see that the understanding level of AI, medical AI, and ophthalmic AI among the medical workers who participated in the survey is higher than that among other professional technicians, suggesting that in China, AI is relatively more widely popularized among medical workers. In recent years, AI has been more and more widely introduced in the medical field [16, 31-34]. Due to work or academic exchanges, medical workers have more opportunities to learn about medical AI than other professional technicians. The other professional technicians in this survey were technical personnel whose research areas had no connection to medicine or AI. As a result, they had relatively less knowledge about medical AI and fewer opportunities to apply or experience medical AI, especially in the more specialized clinical ophthalmology field. Naturally, those in the survey who had no medical background would have a difficult time imagining the application of AI in medicine. Therefore, the real-life application of AI in other areas should be further popularized in our society to gain more of people's trust before AI can be used in medical care.

Concerning the attitudes toward AI, both medical workers and other professional technicians were relatively confident in human doctors, with only a very small proportion of people thinking that AI in ophthalmology would completely replace ophthalmologists. Most
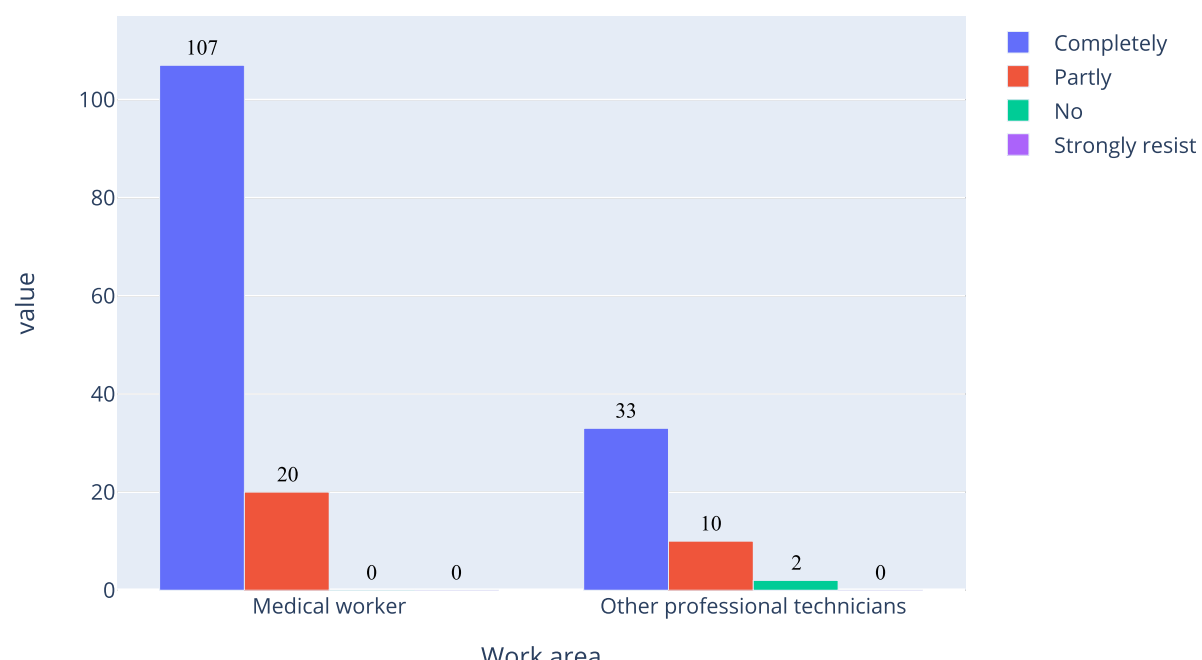

Work area

Fig. 8 Acceptance level of ophthalmic artificial intelligence among the respondents who had experience in ophthalmic artificial intelligence 
Table 6 Simple version of medical workers and other professional technicians ' attitudes of artificial intelligence ( $\mathrm{N}$ of medical workers $=291, \mathrm{~N}$ of other professional technicians $=271$ )

\begin{tabular}{|c|c|c|c|c|c|c|}
\hline Attitude & $\begin{array}{l}\text { Medical } \\
\text { workers, } \mathrm{n}\end{array}$ & $\begin{array}{l}\text { other professional } \\
\text { technicians, } \mathrm{n}\end{array}$ & $\begin{array}{l}\text { Chi-Square, } \\
\mathrm{X} 2\end{array}$ & $\begin{array}{l}\text { Asymp. Sig., } \\
\text { P }\end{array}$ & OR & $95 \% \mathrm{Cl}$ \\
\hline \multicolumn{7}{|l|}{ Al in ophthalmology will replace ophthalmologists } \\
\hline Completely and Partly & 224 & 157 & 23.304 & 0 & 2.428 & $1.687-3.494$ \\
\hline Not and Don't understand & 67 & 114 & & & & \\
\hline \multicolumn{7}{|l|}{ Do you have any experience with Al in ophthalmology? } \\
\hline $\begin{array}{l}\text { Being using or about to use, Having applied and Having } \\
\text { experienced }\end{array}$ & 127 & 45 & 48.299 & 0 & 3.889 & $2.620-5.773$ \\
\hline Having no related experience & 164 & 226 & & & & \\
\hline \multicolumn{7}{|l|}{ Acceptance level of Al in ophthalmology } \\
\hline Completely and Partly & 288 & 262 & 3.522 & 0.061 & 2.357 & $0.883-12.312$ \\
\hline Not accept and Strongly resist & 3 & 9 & & & & \\
\hline
\end{tabular}

respondents were relatively rational, believing that ophthalmic AI would only partly replace ophthalmologists. As people become more rational about AI in ophthalmology, the condition has been more and more favorable for the healthy development of AI in the medical field. As suggested by Turing for the healthy development of AI, "Instead of trying to produce a program to simulate the adult mind, why not rather try to produce one which simulates the child's? If this were then subjected to an appropriate course of education one would obtain the adult brain." [35] This conception has functioned as guidance in the research of medical AI. From JAMA's publication of AI used for DR diagnosis in 2016 [16] to the U.S. Food and Drug Administration's approval of IDx-DR in 2018 [25], there has been no real diagnostic systems that can fully diagnose and identify all the 4 grades of DR, which means there is still a long way for ophthalmic AI to go from laboratory research to clinical application. But undeniably, in a country such as China where ophthalmologists are in much dire need (the over 1.4 billion Chinese people only share about 44,800 ophthalmologists) [36], the application of AI can

Table.7 Respondents' concerns about artificial intelligence ( $N=$ 562)

\begin{tabular}{ll}
$\begin{array}{l}\text { What are your concerns about Al in ophthalmology? } \\
\text { (multiple selection) }\end{array}$ & $\begin{array}{l}\text { Respondents } \\
\mathbf{n}(\%)\end{array}$ \\
\hline Medical responsibilities are unclear & $317(56.4)$ \\
Service price is too expensive & $252(44.8)$ \\
Service quality is difficult to guarantee & $277(49.3)$ \\
Medical ethical risk & $240(42.7)$ \\
Policy support may not be in place & $235(41.8)$ \\
Others & $85(15.1)$ \\
Is it necessary to strengthen the study of & Respondents \\
medical ethics in the ophthalmic Al field? & $n(\%)$ \\
Yes & $517(92.0)$ \\
No & $45(8.0)$ \\
\hline
\end{tabular}

help to diagnose and treat many more patients, which would reduce the burden on ophthalmologists, thus probably having led to the higher acceptance level of ophthalmic AI among medical workers. Meanwhile, those other professional technicians, despite a relatively lower understanding level, also basically held a positive attitude to acknowledging $\mathrm{AI}$ in ophthalmology.

As far as the application of ophthalmic AI goes, there are currently some pilot centers in China that provide opportunities to experience its real-life application. However, due to the rarity of such centers, only close to half of the medical workers involved in the survey had related experience while $4 / 5$ of the other professional technicians had no such experience. This indicates that the application of AI in ophthalmology is not yet popularized. It has very few real-life applications, mainly due to technical and ethical issues. On the technical side, the systems that had relatively good diagnosing abilities turned out to be not well performed in the complex real-life scenarios, requiring further improvement. And on the ethics side, the medical responsibilities are not clearly defined for the artificial intelligence diagnostic systems and there are no related policies to follow or to regulate it with. Therefore, although the research of AI in ophthalmology is going well, more pilot centers are needed to explore the problems that may be encountered before actual application; it still takes prudence in its real-life application.

In recent years, the application of $\mathrm{AI}$ in ophthalmology is very deeply researched, but there are not so many studies on related policies and ethics. The survey found that unclear medical responsibilities and difficulty in guaranteeing service quality respectively ranked as the No. 1 and No. 2 concerns about the use of AI in ophthalmology, with $60 \%$ of the respondents worrying about the "unclear medical responsibilities." These concerns are sufficient to show that the country needs to improve its regulation system of $\mathrm{AI}$ and strengthen the 


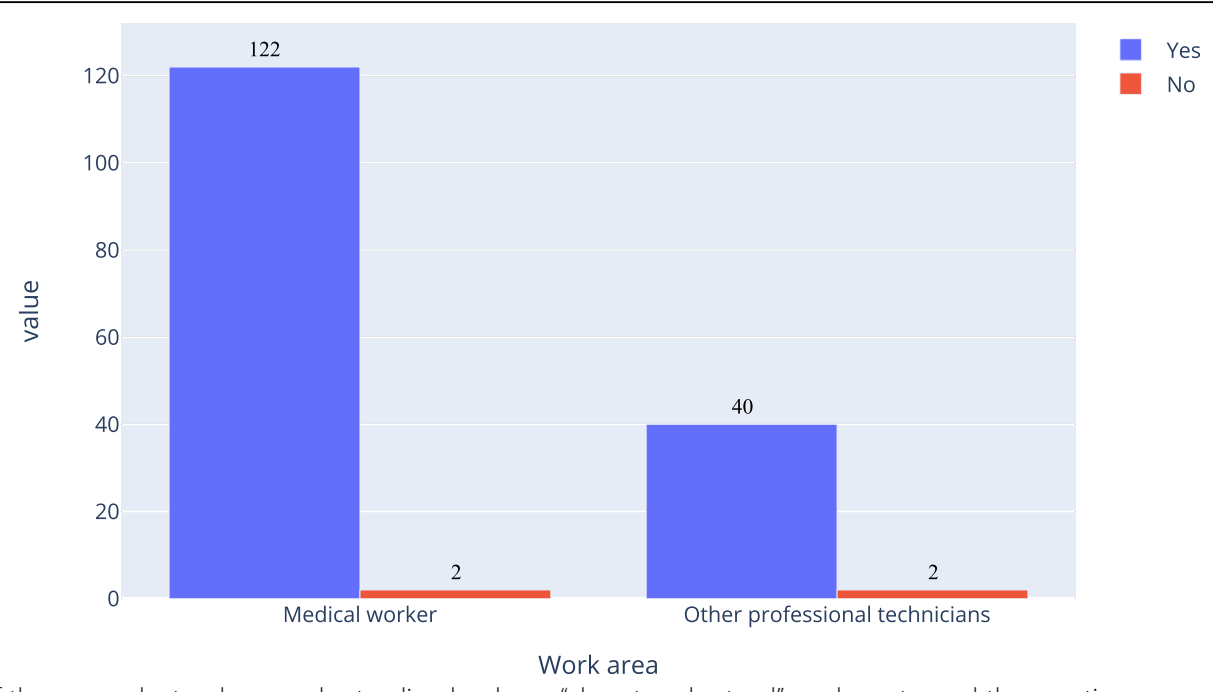

Fig. 9 Attitudes of the respondents whose understanding level was "almost understand" or above toward the question

exploration of relevant medical ethics issues. Only when the relevant regulation system and ethics issues have been addressed can we guarantee the real-life practice of medical AI, and establish people's confidence in medical AI so that they can truly accept its relevant application. Therefore, the next step is to research on AI technology that can eliminate ethical risks and non-technical countermeasures, etc.

The survey mainly analyzed the respondents' understanding and acceptance level of AI in ophthalmology, as well as the respondents' concerns about AI in ophthalmology. The respondents in this survey are mainly from Zhejiang, China, an important part of the Yangtze River Delta, which is one of china's relatively developed regions. With almost no respondents from remote areas, the survey results do not represent those of medical workers and other professional technicians nationwide. Besides, although the respondents are relatively evenly distributed among the different groups, the total number is relatively small. The subjects of the survey are mainly divided into medical workers and other professional technicians. This survey mainly studies the understanding and attitudes of ophthalmology AI in different working fields, and the research angle is relatively single. The follow-up surveys should try to broaden the survey scope and increase the angle of research angle (doctors, patients, etc.), making the findings more credible and broadly representative.

\section{Conclusions}

The survey results revealed that the medical workers had a higher understanding level of AI in ophthalmology than other professional technicians, making it necessary to popularize ophthalmic AI education among other professional technicians. Most of the respondents did not have any experience in ophthalmic AI, but generally had a relatively high acceptance level of AI in ophthalmology, and there was a need to strengthen research into the medical ethics issues of the field. The next step of this research is to expand the scope of survey, increase the angle of survey, and carry out research on AI technology that can eliminate ethical risks and research on non-technical countermeasures,promoting the clinical application of AI.

\section{Abbreviations}

Al: Artificial intelligence; OCT: Optical coherence tomography; DR: Diabetic retinopathy

\section{Acknowledgements}

Not applicable.

\section{Authors' contributions}

During the study, all authors have contributed significantly. WY and BZ, FF analyzed data and drafted the manuscript. MW and $\mathrm{SZ}, \mathrm{HZ}$ analyzed data and revised the draft. WY, BZ, MW, XH, YJ and JW designed electronic questionnaire. $W Y, B Z, M W, X H$, and $X P$ sent the questionnaire to respondents. WY and XP designed the experiment and built initial constructs. WY, BZ and XP together proposed the idea and supervised the project. All authors have read and approved the manuscript.

\section{Funding}

The National Natural Science Foundation of China (61906066) mainly studies vision prediction after cataract surgery. The Natural Science Foundation of Zhejiang Province (LQ18F020002) mainly studies DR images labeling and diagnosis based on it. The Zhejiang Basic Public Welfare Research Program (LGF18H120003) mainly studies intelligent diagnostic systems of DR. The Zhejiang Medical and Health Research Project (2020PY027) and the Huzhou Science and Technology Planning Program (2019GY13) mainly study the cross field application and promotion of artificial intelligence and ophthalmological diagnostic technologies. All the above foundations focus on the cross field application of artificial intelligence in medicine (especially in ophthalmology) and need to know about people's knowledge of artificial intelligence, artificial intelligence in medicine and artificial intelligence in ophthalmology, which is why the support for the study. 


\section{Availability of data and materials}

The data and materials used and/or analyzed during the present study are available from the corresponding author on reasonable request.

\section{Declarations}

\section{Ethics approval and consent to participate}

In China, the National Health and Family Planning Commission officially issued Measures for the Ethical Review of Biomedical Research Involving Humans in 2016. The Article 3 defines the biomedical research involving human subjects.

Article 3. Biomedical research involving human subjects in the Measures includes:

(1) activities of conducting research on human physiology, psychological behavior, pathological phenomena, causes and pathogenesis of diseases, as well as the prevention, diagnosis, treatment and rehabilitation of diseases, using modern methods in physics, chemistry, biology, traditional Chinese medicine and psychology, etc;;

(2) activities of experimental research on the human body of new medical technologies or new medical products;

(3) activities in which scientific research materials such as specimens, medical records and behaviors relating to humans are collected, recorded, used, reported or stored by epidemiological, sociological and psychological methods.

This study mainly investigates people's current understanding of the cross field application of artificial intelligence in medicine, which does not involve the activities stipulated in Article 3 of the censorship measures. Thus ethical review is not required for the study.

Respondents were informed verbally as well as in written form in the questionnaire that completing and submitting the questionnaire meant they agreed to participate in the study.

\section{Consent for publications}

Not Applicable.

\section{Competing interests}

The authors declared no competing interests.

\section{Author details}

${ }^{1}$ School of Information Engineering, Huzhou University, Zhejiang 313000 Huzhou, China. ${ }^{2}$ Zhejiang Province Key Laboratory of Smart Management \& Application of Modern Agricultural Resources, Huzhou University, 313000 Huzhou, China, Zhejiang Province. ${ }^{3}$ College of Computer and Information, Hehai University, 210013 Nanjing, China, Jiangsu. ${ }^{4}$ Department of Endocrinology, First Affiliated Hospital of Huzhou University, 313000 Huzhou, China, Zhejiang. ${ }^{5}$ School of Medicine, Huzhou University, 313000 Huzhou, China, Zhejiang. ${ }^{6}$ Zhejiang University Real Doctor Al Research Center, 310000 Hangzhou, Zhejiang, P.R. China. ${ }^{7}$ Affiliated Eye Hospital of Nanjing Medical University, No.138 Hanzhong Road, Gulou District, 210029 Nanjing, Jiangsu, China. ${ }^{8}$ First People's Hospital of Huzhou, 313000 Huzhou, China, Zhejiang.

Received: 17 December 2020 Accepted: 17 September 2021 Published online: 09 October 2021

\section{References}

1. KrizhevskyA,Sutskeverl,HintonG E.Imagenet classification with deep convolutional neural networks[C]//Advances in neural information processing systems.2012:1097-1105.

2. SimonyanK,ZissermanA.Verydeepconvolutionalnetworksforlargescaleimagerecognition[C]//

AdvancesinInternationalConferenceonLearningRepresentations.May2015.

3. SzegedyC,LiuW,JiaY,etal.Goingdeeperwithconvolutions[C]// ProceedingsofthelEEEconferenceoncomputervisionandpatternrecognition. 2015:1-9.

4. HeK,ZhangX,RenS,etal.Deepresiduallearningforimagerecognition[C]// ProceedingsofthelEEEconferenceoncomputervisionandpatternrecognition. 2016:770-778

5. HuangG,LiuZ,Van DerMaatenL,etal. Denselyconnectedconvolutionalnetworks[C]// ProceedingsofthelEEEconferenceoncomputervisionandpatternrecognition. 2017:4700-4708.
6. GirshickR,DonahueJ,DarrellT,etal.Richfeature hierarchiesforaccurateobjectdetectionandsemanticsegmentation[C]// ProceedingsofthelEEEconferenceoncomputervisionandpatternrecognition. 2014:580-587.

7. RedmonJ,DivvalaS,GirshickR,etal.Youonlylookonce:Unified,realtimeobjectdetection[C]//

ProceedingsofthelEEEconferenceoncomputervisionandpatternrecognition. 2016:779-788.

8. LongJ,ShelhamerE,DarrellT.

Fullyconvolutionalnetworksforsemanticsegmentation[C]//

ProceedingsofthelEEEconferenceoncomputervisionandpatternrecognition. 2015:3431-3440.

9. RonnebergerO,FischerP,BroxT.U-net:

Convolutionalnetworksforbiomedicalimagesegmentation[C]// InternationalConferenceonMedicalimagecomputingandcomputerassistedintervention.Springer,Cham,2015:234-241.

10. XingjianS H I,ChenZ,WangH,etal.Convolutional LSTM network: a machine learning approach for precipitation nowcasting[C]//Advances in Neural Information Processing Systems.2015:802-810.

11. Esteva A, Kuprel B, Novoa RA, et al. Dermatologist-level Classification of Skin Cancer with Deep Neural Networks[J]. Nature; 2017: 115-118.

12. Orringer DA, Pandian B, Niknafs YS, et al. Rapid intraoperative histology of unprocessed surgical specimens via fibre-laser-based stimulated raman scattering microscopy[J]. Nature Boimedical Engineering; 2017: 1-13.

13. Liang $\mathrm{H}$, Tsui BY, Ni H, et al. Evaluation and accurate diagnoses of pediatric diseases using artificial intelligence[J]. Nat Med. 2019;25(2):433-8.

14. Gurovich $Y$, Hanani $Y$, Bar $O$, et al. Identifying facial phenotypes of genetic disorders using deep learning[J]. Nat Med. 2019;25(1):60-4.

15. Yeung S, Rinaldo F, Jopling J, et al. A computer vision system for deep learning-based detection of patient mobilization activities in the ICU[J]. npj Digital Medicine; 2019;3:1-5.

16. Gulshan V, Peng L, Coram M, et al. Development and validation of a aeep learning algorithm for detection of diabetic retinopathy in retinal fundus photographs[J]. JAMA. 2016;316(22):2402-10.

17. Poplin R, Varadarajan AV, Blumer K, et al. Prediction of cardiovascular risk factors from retinal fundus photographs via deep learning[J]. Nature Biomedical Engineering. 2018;2(3):158-64.

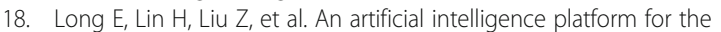
multihospital collaborative management of congenital cataracts[J]. Nature Biomedical Engineering. 2017;1(2):1-8.

19. Kermany S,Goldbaum M,Cai W. Identifying medical diagnoses and treatable diseases by image-based deep learning[J].Cell;2018,172(5):1122-1131.

20. Pekala M,Joshi N,Liu T Y A. Deep learning based retinal OCT segmentation[J].Computers in Biology and Medicine;2019,114:103445.

21. GaoK,NiuS,JiZ,etal.Double-branched and area-constraint fully convolutional networks for automated serous retinal detachment segmentation in SDOCT images[J].Computer Methods and Programs in Biomedicine;2019,176: 69-80.

22. ZhangH,NiuK,XiongY,etal.Automatic cataract grading methods based on deep learning[J].Computer Methods and Programs in Biomedicine;2019,182:104978.

23. de LaTorreJ,VallsA,PuigD.A deep learning interpretable classifier for diabetic retinopathy disease grading[J].Neurocomputing;2019.

24. ZhaoH,SunY,LiH.Retinal vascular junction detection and classification via deep neural networks[J].Computer Methods and Programs in Biomedicine; 2020,183:105096.

25. Abràmoff MD, Lavin PT, Michele $\mathrm{B}$, et al. Pivotal trial of an autonomous Albased diagnostic system for detection of diabetic retinopathy in primary care offices[J]. npj Digital Medicine; 2018, 1(1): 1-8.

26. Dos Santos DP, Giese D, Brodehl S, et al. Medical students' attitude towards artificial intelligence: a multicentre survey[J]. European Radiology; 2019, 29(4): pp. 1640-6.

27. Abid S, Awan B, Ismail T, et al. Artificial intelligence: medical students' attitude in district Peshawar Pakistan[J]. Pakistan Journal of Public Health. 2019;9(1):19-21.

28. Gong B, Nugent JP, Guest W, et al. Influence of artificial intelligence on Canadian medical students' preference for radiology specialty: a national survey study[J]. Academic Radiology; 2019, 26(4): pp. 566-77.

29. Vayena E, Blasimme A, Cohen IG. Machine learning in medicine: Addressing ethical challenges[J]. PLoS Med. 2018;15(11):e1002689.

30. Keskinbora $\mathrm{KH}$. Medical ethics considerations on artificial intelligence[J]. Journal of Clinical Neuroscience; 2019. 
31. Yang WH, Zheng B, Wu MN, et al. An Evaluation System of Fundus Photograph-Based Intelligent Diagnostic Technology for Diabetic Retinopathy and Applicability for Research[J]. Diabetes Therapy; 2019, 10(5).

32. Xu J, Yang W, Wan C, et al. Weakly supervised detection of central serous chorioretinopathy based on local binary patterns and discrete wavelet transform[J]. Comput Biol Med. 2020;127:104056.

33. Lin X, Fu Y, Li L, et al. A Novel Quantitative Index of Meibomian Gland Dysfunction, the Meibomian Gland Tortuosity[]]. Translational Vision Science, Technology; 2020, 9(9).

34. Turing AM. Computing machinery and intelligence[M]//Parsing the Turing Test. Dordrecht: Springer; 2009. pp. 23-65.

35. Propaganda Department. Transcript of the regular press conference of the National Health Commission on June 5, 2020[EB/OL]. [2020-6-5]. http:// www.nhc.gov.cn/xcs/s3574/202006/1f519d91873948d88a77a35a427c3944. shtml. 2020.6.5.

36. Emma Beede. 2020. Healthcare Alsystems that put people at the center[EB/ OL].[2020-4-25]. https:/www.blog.google/technology/health/healthcare-aisystems-put-people-center/.

\section{Publisher's Note}

Springer Nature remains neutral with regard to jurisdictional claims in published maps and institutional affiliations.

- fast, convenient online submission

- thorough peer review by experienced researchers in your field

- rapid publication on acceptance

- support for research data, including large and complex data types

- gold Open Access which fosters wider collaboration and increased citations

- maximum visibility for your research: over $100 \mathrm{M}$ website views per year

At $\mathrm{BMC}$, research is always in progress. 